\title{
LA FILOSOFÍA Y LA CULTURA ANTE LA GLOBALIZACIÓN
}

\author{
Alejandro Serrano Caldera*
}

\section{RESUMEN}

La globalización ha producido una crisis de valores y un riesgo a la pluralidad cultural. Frente a la crisis integral de nuestro tiempo, la filosofía debe contribuir a forjar una ética de los valores, fundada en una racionalidad moral y conceptual que enfrente y sustituya a la racionalidad instrumental.La filosofía debe contribuir a construir un mundo más humano, donde la tecnología esté al servicio de los valores y no los valores y el ser humano al servicio de los instrumentos de dominación y de poder político universal, para el que los Estados-Naciones no son más que correas de transmisión de una sola voluntad de dominación.

Palabras clave: Globalización. Filosofía. Cultura. Etica. Valores

\begin{abstract}
PHILOSOPHY AND CULTURE IN THE FACE OF GLOBALIZATION

Globalization has produced a crisis of values and a risk to cultural plurality. In the face of the generalized crisis of our time, philosophy must help drawing up an ethic of values, based on a moral and conceptual rationality to face and replace the instrumental rationality. Philosophy must contribute to building a more humane world, where technology serves values and not values and the human being serve the instruments of domination and universal political power for which Nation-States are merely conduits for a single will to dominate.
\end{abstract}

Keywords: Globalization. Philosophy. Culture. Ethics. Values.

La globalización consiste en una práctica y un concepto que pretende convalidar un modelo homogéneo, a partir del cual se van generando las diferentes manifestaciones de la historia. Se trata de un modelo único de sociedad, de un arquetipo universal que pretende ser, en este momento, la forma de todas las sociedades cualquiera sea su naturaleza, historia o identidad.

Este problema de la globalización se ve a través de la generalización de determinadas categorías económicas, políticas y sociales y de los efectos producidos por una aceleración vertiginosa en los cambios de la tecnología. Categorías y realidades históricas que han sido fundamentales para la Modernidad como las de política, soberanía, Estado-Nación, para referirme a las que nos son más próximas, se ven afectadas por un concepto y una práctica central que pretende legitimarse en la idea de la globalidad. Este modelo diseñado en los centros de dominación mundial, se asume valido para cualquier sociedad en cualquier parte del mundo.

\footnotetext{
* Doctor en Derecho, Universidad Nacional Autónoma de Nicaragua, 1962. Profesor de diversas cátedras de grado y maestrías en la Universidad Americana, UAM; Universidad Nacional Autónoma de Nicaragua, UNAN-Managua y Universidad Centroamericana, UCA.Endereço para correspondência: Mansión Teodolinda, 6 cuadras al Sur, 25 varas abajo, Bolonia, Managua, Nicaragua. Tel: (505) 226621 28. E-mail: alejandro.serrano@turbonett.com.ni
} 
La globalización es una realidad y como tal debe considerarse. El rechazo pasional es estéril; el silencio indiferente, cómplice. Lo importante es tratar de señalar racionalmente que un proceso de esta naturaleza no debe escapar a una moral exterior que le sirva de referencia, ni a un juicio de valor que deba justificarla. Las cosas no se justifican por el solo hecho de existir. Hay que ver como existen y para qué.

La globalización es necesaria siempre que se la entienda como unidad en la diversidad. Si ha de existir una cultura universal compatible con la dignidad e identidad humanas, ésta debe ser el resultado de la síntesis de múltiples afluentes culturales y expresiones históricas. Si una cultura planetaria llegara a surgir como consecuencia de ese abrazo universal de las culturas, de ese diálogo de las identidades, bienvenida sea. Si en cambio, debemos disolvernos sin rostro en un modelo unilateral, que además, no siempre representa una cultura superior, sino, con frecuencia, los residuos de una subcultura, que no es mejor, sino que solamente proviene de una sociedad más poderosa, tenemos la obligación de resistir y luchar para que una verdadera universalidad, producto de la multiculturalidad, florezca.

El concepto de globalización, como ha sido formulado y aplicado, es lo contrario del de unidad en la diversidad. Incluye la existencia de un paradigma y la propuesta de un mundo homogéneo. En este sentido, globalización equivale a uniformidad, pero no a igualdad, ni siquiera a proporcionalidad, pues su esencia es, precisamente, la desigualdad y la asimetría entre los diferentes componentes del sistema.

En la base misma del poder del sistema se encuentran, los Estados y las corporaciones transnacionales, verdadero núcleo de todo el mecanismo.

Entre ambos, Estados y corporaciones, hay una identidad de intereses y fines, pues son piezas complementarias de la estructura principal y están situados en la búsqueda de sus objetivos, en un mismo o muy cercano plano jerárquico. En cambio, los Estados de los países del llamado mundo subdesarrollado son piezas secundarias en el engranaje del mecanismo.

La globalización se nos presenta así como un mecanismo esencialmente asimétrico, compuesto de una parte por las piezas claves que conforman los centros vitales de planificación, decisión y distribución de funciones: las corporaciones trasnacionales, los Estados del núcleo de poder mundial y los organismos financieros encargados de formular y aplicar las políticas correspondientes. De otra parte, las sociedades periféricas, caracterizadas, en medio de sus diferencias, por algunos referentes comunes: economías dependientes, escasa o nula industrialización, retraso en la utilización de la tecnología de punta, inestabilidad política, fragilidad democrática, debilidad institucional, concepto, estructura y funcionamiento anacrónicos del Estado, masificación de la pobreza crítica, crisis de sus sistemas educativos, para mencionar algunos rasgos que podrían considerarse comunes a pesar de sus diferentes grados de incidencia.

Entre estos dos rangos que conforman el sistema no existe una estructura coherente, pues los dos términos no son jerarquizables por pertenecer a configuraciones cualitativamente diferentes. El sistema mundial, que se expresa en la globalización, se construye a partir de un modelo único que no toma en cuenta características e identidades de los componentes periféricos los que por su naturaleza y situación son colaterales.

¿Quiero esto decir que la globalización es mala y la integración dañina? Depende. Lo sería, si la globalización, de cualquier signo que sea, se logra restringiendo o anulando las posibilidades de otras expresiones culturales; es decir, si lo homogéneo no es síntesis de diferentes manifestaciones, si no es un nivel que se alcanza mediante un progresivo escalonamiento que incluye las realidades nacionales y regionales.

Puesto en términos positivos quiere decir que la integración, y la globalización, pueden ser beneficiosas, si se realizan a partir del encadenamiento de situaciones y de la estructuración de propuestas y alternativas que se van construyendo escalonadamente. Expresado en forma más directa y precisa, el desafío actual para nuestros pueblos es el de construir planes estratégicos, nacionales, subregionales y regionales, que den nuevo contenido a los procesos de globalización, que permitan rescatar la circunstancia en la que vivimos y en la que se forman el destino personal de cada quién y la historia de cada pueblo. 
Para nosotros, herederos de la pobreza mundial, pero también de un universo cultural antiguo y polivalente, es de ineludible obligación plantearnos con lucidez el problema, sin beaterías de izquierda ni de derecha que se resisten a pensar con libertad sometidas a los dogmas; que satanizan o sacralizan y niegan la oportunidad de irrupción de un pensamiento crítico, a cuya ausencia se deben casi todos nuestros males.

La globalización no es en sí misma un mal o un bien. Será lo primero, si prevalecen los criterios que pretenden una sociedad uniformada. Será lo segundo, si la globalización equivale a una verdadera universalidad, formada por la conjunción de las más variadas expresiones de la creatividad humana.

La globalización y robotización de los fenómenos económicos, la transnacionalización de la economía y de los procesos financieros, vienen determinando una restricción a lo que ha constituido la esencia del Estado moderno: la soberanía.

Las realidades que en el campo político han servido de base a la Modernidad, están afectadas por el concepto y el fenómeno de la globalidad. Pero además de este hecho económico y financiero, y de la existencia de un modelo económico transnacional y global, se da un proceso de tecnologización acelerada que produce también sus propias consecuencias.

Es el caso de los avances tecnológicos, de las redes que constituyen hoy por hoy una especie de realidad sobrepuesta al mundo que nosotros conocemos y definimos con sus posibilidades y sus límites. Es el sistema de redes de comunicación que crean la posibilidad de una realidad y un lenguaje universal. Esto nos plantea un enorme desafío político, cultural y teórico, en la medida en que este sistema ofrece, como nunca antes, a la par de inmensas oportunidades de integración y desarrollo, las posibilidades de un dominio total ejercido por un poder planetario.

Están dadas las condiciones y elaborados los instrumentos para establecer una civilización planetaria. Está por verse si ésta será fruto del diálogo y retroalimentación de las culturas, o si será consecuencia de la implantación de un modelo en el que los medios se habrán transformado en fines produciéndose la inversión teleológica de la que nos hablaba Hegel a comienzos del siglo XIX.
El desafío está en definir la manera conforme a la cual debemos integrarnos a estos sistemas, contribuyendo a la formación de una civilización planetaria que sea fruto de la unidad en la diversi$\mathrm{dad}$, neutralizando los riesgos de transformarnos en sólo consumidores de mensajes estandarizados que erosionan nuestra identidad y valores y transforman a la civilización en un engranaje de la uniformidad.

El desarrollo del ser humano, de sus aptitudes y capacidades, se forma a raíz del proceso de apropiación de la cultura, creada por medio del trabajo a través de la historia.

Esto es un proceso activo, pues supone, no sólo la asimilación de la cultura acumulada, sino además, el desarrollo de actividades específicas en el hombre, en virtud de la apropiación que hace de los objetos sociales.

La transferencia cultural y tecnológica en las sociedades dependientes no reproduce ni asimila socialmente los instrumentos transferidos. La adaptación es mecánica y por lo mismo no genera un proceso cultural en sentido dinámico e histórico. Por el contrario, estos mecanismos culturales producen actitudes pasivas que a la larga llevan a la incapacidad creativa y generan, o más bien degeneran en una conducta de sometimiento mecánico.

El ser humano ajeno al proceso de producción de la cultura se convierte en un prisionero de ella. La enajenación de la cultura produce el efecto inverso que la creación de la cultura. Mientras ésta por el trabajo produce al mundo y produce al hombre alejándolo de las leyes biológicas e incorporándolo a la vida histórico-social, aquella, la cultura enajenada, suprime la capacidad de creación, y la asimilación histórica y la vocación de cambio, que son los rasgos esenciales del ser humano.

La cultura es el mundo vivencial del ser humano. Pero no toda cultura es auténtica. La inautenticidad de la cultura está ligada a la crisis del humanismo. Es auténtica, cuando al mismo tiempo que hace progresar al hombre lo hace cada vez más humano. Cuando en el remoto amanecer de la especie lo rescata de la zoología a la historia. Cuando lo reafirma cada vez más en sus signos de humanidad, como ser libre, creativo, productor y feliz. Es inauténtica, cuando aún haciéndolo progresar materialmente, lo niega como tal, al reducirlo pasivamente en sus redes, al regresarlo de 
la historia que es proceso de creación del mundo y de auto producción del hombre, a las orillas de la vida biológica y de la naturaleza vegetal, aunque este hecho ocurra en la era de las computadoras, la cibernética y la conquista de universo.

La cultura como tal, en sentido humanista y auténtico, es el proceso de creación, asimilación y recreación del mundo, para el bienestar, la libertad y la auto producción del hombre. Esta, sin embargo, deshumaniza y esclaviza, cuando el modo de producción persigue solamente el lucro, la riqueza y el beneficio, y se sustenta en la explotación de los hombres y de los pueblos. Cuando "la concentración de la riqueza material produce la concentración de la riqueza espiritual". Cuando los valores de un sector preponderante, desde el punto de vista de la economía, se internalizan abstractamente como valores de toda la sociedad. Cuando las diferentes situaciones económicas, sociales y culturales, rompen "la unidad de la especie" y generan condiciones de dependencia interna y externa. Cuando en el campo cultural, y al igual que en otros campos, el mundo se divide en dos: los que producen la cultura y los que en el mejor de los casos se adaptan, o se mal adaptan a la cultura producida por otros. Es decir los que viven al margen de la cultura, imposibilitados, y, luego, incapacitados de crear su propio hábitat, de modificar su medio y en disposición de adaptación sin posibilidades ni intenciones creativas. Cuando se acepta sin análisis crítico y sin racionalización, la cultura y la tecnología de otras sociedades, sin llegar a comprender el proceso científico de su producción ni su necesidad histórica.

Este es el gran riesgo y el drama de nuestro tiempo. Por una parte, seres enajenados culturalmente y caracterizados por una vida pasiva y refleja, seres que ven al mundo frente a un espejo hasta llegar a ser incapaces de conocer y concebir otra realidad que no sea el reflejo de la imagen. Pueblos con marcado subdesarrollo económico, social, científico y técnico, colocados al borde del abismo de la historia, ante la grave posibilidad que el desarrollo científico y técnico ahonde aún más las grietas que lo separan del mundo desarrollado y lo dañen irreversiblemente.

La transposición de esa realidad, a un medio que técnicamente no ha alcanzado tales niveles de desarrollo, crea en éste, necesidades externas y origina una fractura entre el grado de desarrollo interno y la necesidad producida en forma externa. En virtud de esa disociación, la cultura tecnológica incorporada en los objetos, no es asimilada activamente y por ello genera una conducta pasiva de adaptación y luego de sometimiento a los objetos y a quienes los producen.

Es claro pues, en cualquiera de las dimensiones en que se enfoque el asunto, que el problema esencial de nuestro tiempo es la pérdida de la libertad, y por ello, la deshumanización colectiva, como segregado de la forma de vida de la sociedad contemporánea. El hombre, cegado por la ambición de acumular riquezas, o abrumado por los problemas materiales, de supervivencia, está limitado para concebir el sentido de la libertad más allá del tejido de la realidad socio-económica en la que se encuentra atrapado.

La lucha por la libertad es, en primera instancia la expresión del derecho de la supervivencia biológica. Esta actitud legítima, aún y cuando es condición necesaria para que aflore la libertad, pues ésta no existe en la explotación y en la miseria, no agota en ese nivel la plenitud a la que el ser humano tiene derecho.

El riesgo para la cultura es muy grande, pues estamos enfrentados a un desafío que puede permitirnos desarrollar de manera extraordinaria los verdaderos valores universales, dentro de los cuales están incluidos los propios, o perecer culturalmente en la avalancha de una tecnificación que no se detiene ante la identidad de las culturas ni ante las diferencias.

Estamos ante un proceso de globalización no sólo de la economía, de transnacionalización no sólo de los mecanismos financieros, sino de globalización y transnacionalización de los modelos sociales, políticos y culturales que de alguna forma se van transmitiendo como paradigmas de la comunidad humana.

La supranacionalización del capital y la transnacionalización de la producción están modificando los conceptos Norte y Sur basados originalmente en la relación entre la geografía, las condiciones económicas y sociales y la división internacional del trabajo, por un nuevo concepto en el que las fronteras se mueven y la geografía cambia. En numerosas 
ocasiones hemos señalado de qué manera, con la transnacionalización de la producción, la riqueza y la pobreza devienen fenómenos supranacionales en una geografía que se va adaptando a los requerimientos de un sistema con pretensiones de cobertura planetaria.

Frente a la razón instrumental que constituye la lógica de este proceso de transnacionalización de la producción, es nuestro deber trabajar en la elaboración de una filosofía moral que humanice ese alucinante proceso y que descodifique los signos en clave que la realidad económica, política, social y cultural postindustrial conllevan. Lo primero es desmontar la identidad que se trata de establecer entre transnacionalización, globalización y uniformidad con universalidad. Globalidad no es universalidad; lo homogéneo no es universal, pues la homogeneidad se logra restringiendo o anulando las posibilidades de otras expresiones culturales. Lo que uniforma no une, somete.

Lo verdaderamente universal en la cultura es lo que unifica en su propia heterogeneidad dentro de una articulación determinada que permite no sólo que las culturas diferentes coexistan, sino también que sean capaces de retroalimentarse. Esta es una de las labores inmediatas a desarrollar: construir una ética de la racionalidad, del desarrollo y de la democracia, para adaptar críticamente los sistemas tecnológicos en forma tal que pueda aprovecharse lo mejor que conlleva la maravillosa experiencia de la ciencia y la tecnología y, a la vez, evitar que una transferencia cultural acrítica e inconsciente nos conduzca, en un tiempo no demasiado largo, a la abolición de nuestro propio rostro y de nuestro propio rastro.

Una ética de los valores exige desde el principio señalar la crisis moral que, en buena parte, se debe a la pérdida considerable de autenticidad y a la adopción mecánica de los paradigmas de la sociedad de consumo que se perfilan detrás de la deconstrucción y fragmentación de los arquetipos de la Modernidad. Parte de esta crisis, es como dice Bonete Perales (1989, p. 277), "vivir con las pautas económico-morales de la sociedad de consumo, sin haber llegado a la economía de consumo".

Es imprescindible, de acuerdo con el mismo autor, recuperar la actitud moral partiendo de los problemas concretos como el de la injusticia en el mundo, las desigualdades económicas, el problema de la guerra, el de la violencia, el problema de la corresponsabilidad de los hombres de ciencia, de los intelectuales, de las universidades, etc. Es necesario no dejar la responsabilidad moral a la tecnocracia. (BONETE PERALES, 1989, p. 289-290).

Es inmoral adoptar, o simplemente aceptar de modo pasivo, la lógica del consumo por el consumo en la que, como dice Aranguren (1965 apud BONETE PERALES, 1989, p. 276), "el despilfarro es exaltado psicológicamente como símbolo de pertenencia a un status superior, signo de ascenso social, éxito y triunfo. Del puritanismo se ha pasado al hedonismo, a la moral del puro bienestar, que se hace consistir en el mayor consumo posible de todos los bienes posibles".

Por su parte, una ética de la democracia, agrega Aranguren (1965 apud BONETE PERALES,1989, p. 293-294), indica que

la democracia no es un status en el que puede un pueblo cómodamente instalarse. Es una conquista ético-política de cada día, que sólo a través de una autocrítica siempre vigilante puede mantenerse, como decía Kant de la moral en general, una tarea infinita en la que si no se progresa se retrocede, pues incluso lo ya ganado ha de reconquistarse cada día [...] La democracia nunca puede dejar de ser lucha por la democracia $[\ldots]$ Antes y más profundamente que un sistema de gobierno es un sistema de valores que demanda una educación político-moral [...] El intelectual deberá ejercer una función moralizadora, crítica, utópica y heterodoxa respecto a la democracia establecida.

Al identificar la crisis que enfrentamos, es importante descodificar el neoliberalismo y las teorías del fin de la historia, hacer una crítica al concepto de globalidad, fundamentar el concepto de universalidad a partir de la existencia de las diferencias y fundamentar una nueva ética sobre la base del reconocimiento del otro. En eso reside la clave de una formulación ética para nuestro tiempo: reconocer al otro, lo diferente; reconocer que existen culturas que no necesariamente coinciden, sino que difieren en su riqueza y multivocidad con las culturas hegemónicas que tratan de dar una forma unilineal al mundo contemporáneo. 
En la medida en que seamos capaces de generar esas alternativas filosóficas y éticas, estaremos preparándonos para insertarnos en este proceso de transformación tecnológica y cambios cualitativos que produce una revolución sin precedentes en la historia de la humanidad. En los últimos años se han dado transformaciones profundas. La filosofía no puede dejar pasar estos acontecimientos, ni dejar de relacionar la crisis de la ética, con el vertiginoso desarrollo de la tecnología, el cual se produce desprovisto de los necesarios sustentos morales y conceptuales, ya que es justamente la unión de una teoría y de una práctica lo que puede permitir al mundo desembocar en una experiencia moralmente enriquecedora a través del uso racional y humano de medios tecnológicos que tienen, asimismo, una posibilidad de utilidad sin precedentes.

Es imprescindible, por tanto, ir a las raíces y, en nuestro caso, rescatar los elementos que nos permitan construir esa filosofía moral y esa ética del desarrollo y la democracia para forjar la nueva racionalidad que enfrente a la racionalidad instrumental, la que constituye la lógica dominante de nuestro tiempo. No se puede aceptar un mundo robotizado. Queremos un mundo humano, donde la técnica esté al servicio de los valores y no los valores y el ser humano al servicio de los instrumentos de dominación y de poder político universal, para el que los estados-naciones no son más que correas de transmisión de una sola política de dominación.

Si no somos capaces de hacer una formulación clara de los riesgos que conlleva la transnacionalización económica y política postmoderna y postindustrial, si no somos conscientes de la necesidad de asumir con sentido crítico desde la plataforma de nuestra propia cultura la idea y el proceso del desarrollo contemporáneo, estaremos asistiendo a la sepultura de las culturas, de las diferencias y de las identidades.

Se trata de preservar la identidad histórica y la pervivencia y acción recíproca de todas las culturas; de forjar un concepto de universalidad a través del diálogo de las culturas y de la unidad en la diversidad. Para ello hay que sustituir el juego de una sola imagen y de espejos múltiples por un concepto y una práctica de integración y retroalimentación de todas las historias y todas las culturas. No debemos aceptar ser los espejos en que se multiplica la imagen del poder absoluto que se mira en ellos como Narciso en el estanque.

Es importante que seamos capaces de reivindicar el reconocimiento del otro reafirmando nuestras culturas y planteando la ética de la alteridad, del respeto a las diferencias y de la solidaridad; que seamos capaces de proponer una nueva axiología, una nueva filosofía política que entienda la política como el arte del bien común y que se dirija a la identificación y fortalecimiento de nuevos sujetos históricos de la sociedad civil. Esta filosofía debe revisar la vigencia de los actuales conceptos de política, soberanía, Estado, nación, entendida esta última como proyecto cultural, moral y humano, abierto a una verdadera universalidad, frente a la globalización uniformadora del neoliberalismo.

\section{La filosofía latinoamericana ante la globalización}

La filosofía latinoamericana, desde su propia situación espacio-temporal, desde su historia y su geografía, debe enfrentar el reto del presente. Para ello es imprescindible, de previo, apropiarse teóricamente del pensamiento, la cultura y la historia de América Latina, tener una visión global de la historia de las ideas en la región, con el objeto de reivindicar para ellas la universalidad que les corresponde y para sustentarse sobre ellas, como sobre una plataforma teórica y moral, con el fin de enfrentar los retos del momento actual. Es necesario reiterar esa doble necesidad: la de apropiarnos de nuestra historia de las ideas y la de trascenderla necesariamente al abrirnos, con ella, al desafío de un horizonte más ancho. No hacer cualquiera de las dos cosas señaladas nos llevaría, en un caso, a la abstracción y al vacío, y en el otro, al enclaustramiento y autocolonización.

Es fundamental filosofar sobre este tiempo desde la propia situación espacio-temporal. El desafío que se impone no es sólo pensar nuestra historia, sino, desde ella, pensar la historia de la humanidad.

En virtud de esa circunstancia mediante la cual los contenidos del futuro se encuentran en germen en el presente, es posible entrever, la figura borrosa y todavía latente de las posibilidades del porvenir. Que lo latente se haga patente, que lo ambiguo e incierto se vuelva evidente, que lo posible devenga 
realidad, dependerá de la combinación de una serie de factores sobre los cuales el ser humano tiene la posibilidad de actuar.

No existe sobre los pueblos y su historia un destino inflexible que no pueda ser cambiado mediante la combinación de una visión adecuada y una acción oportuna. No hay un determinismo hermético que consagre a unos como dominadores eternos y condene a otros como dominados perpetuos, pese a que en ciertas circunstancias, como las actuales, parecieran agotadas las posibilidades de cambio, consagrado un arquetipo universal y condenados a la uniformidad total de un solo modelo que suprime las diferencias y reproduce infinitamente su imagen reflejada en infinito número de espejos, reproductores de una visión uniforme y única.

A pesar de todo, un observador medianamente atento puede identificar los cambios, las paradojas y las contradicciones de lo que pareciera ser la instauración del único modelo posible, y llegar a la conclusión de que lejos del fin de la historia nos encontramos más bien en las fronteras de la Modernidad, detrás de las cuales se abren nuevos horizontes.

La Modernidad que se inicia en el siglo XVII se afianza en la racionalidad y en la libertad mediante las cuales, y por medio de la ciencia y la técnica que construyen, el ser humano se lanza a la aventura de forjar su propio mundo como obra de su voluntad y destreza. “¿Quién dudará de que la emancipación humana sólo puede empezar en el momento en que nos asumimos como actores de nuestra propia historia? [...] La racionalización de las relaciones sociales es la característica más importante del paso de las sociedades tradicionales a las modernas" (VILLORO, 1992, p. 95-96).

Junto a esto, agrego, la afirmación de la libertad individual, de la soberanía y del Estado-Nación, en un plano histórico-institucional. Sin embargo, estos valores cambiaron con la consolidación del capitalismo. "Para la Organización burocrática de las sociedades desarrolladas todos los individuos son homogéneos, intercambiables, esclavos de los pequeños intereses personales, computables para las encuestas electorales o los pronósticos del mercado" (VILLORO, 1992, p. 98-99).

Hemos llegado más bien a la frontera de la modernidad, de esa modernidad construida sobre la libertad, la soberanía, el Estado-Nación y la razón histórica. La libertad se pierde desde el momento en que el ser humano deviene un número, un objeto intercambiable; la identidad, cuando el sujeto es estandarizado; la soberanía y el Estado-Nación se diluyen cuando las decisiones dependen cada vez menos de un poder soberano y nacional, para devenir decisiones sin rostro adoptadas por un sistema transnacional que se sobrepone a cualquier interés genuinamente nacional. Es el reino de la razón instrumental, de la deshumanización y de la uniformidad.

Pero, ¿es éste un destino inexorable? ¿No queda ninguna posibilidad para la libertad, la pluralidad y el derecho a la diferencia? Pienso que no es un destino inexorable y que sí hay posibilidad para reivindicar la libertad esencial al ser humano.

Lejos de terminar la historia, creo más bien que es un cierto tipo de historia el que ha entrado en crisis a pesar de las apariencias. Puedo decir con Zea (1993, p. 386) "que se ha iniciado una segunda etapa de la historia universal, la de la realización de la libertad como expresión propia del hombre sin rebajamientos que aplacen su posibilidad. No es así el fin de la historia, sino el auténtico inicio de la historia" en la cual, considero, deberá ser también universal la idea y la práctica de la libertad.

\section{Aproximaciones a un proyecto filosófi- co. Algunas reflexiones sobre el huma- nismo de nuestro tiempo}

El humanismo de nuestro tiempo debe rectificar, reintegrar la unidad fracturada y devolver al hombre y a la mujer su plenitud como seres integrales, intuitivos y racionales, y no como sujetos parciales que han fundado la vida únicamente en la mitad racional del ser. Jano tiene dos rostros y ambos forman su unidad.

La integración dialéctica de ambas tendencias, o lo que es lo mismo, la realización de la unidad en la diversidad de las dos formas de interpretar y actuar la historia y la naturaleza humana, es lo que puede permitirnos "humanizar la vida y vitalizar las humanidades" y así recuperar valores que transcienden la utilidad, el provecho y la acumulación, de los que está lleno el lenguaje, la conducta y las categorías morales de nuestro tiempo. El egoísmo predominante debe dar paso a la solidaridad. Exis- 
tir es una palabra que nos sugiere vivir para algo más que para sí mismo. Ex-sí, fuera de sí, hacia los otros, hacia el prójimo, que es el próximo, es algo más que sobrevivir, es vivir, es más que vivir.

Hablar de un proyecto filosófico desde América Latina exige aclarar que todo proyecto implica una propuesta y toda propuesta es un intento de abrir caminos cuando se considera que otros están cerrados.

El proyecto de la Ilustración que en la filosofía, el derecho y la política, se abre en Europa en el siglo XVIII, lo mismo que el proyecto que propuso el romanticismo y el positivismo en el siglo XIX, parecieran agotados, o al menos en crisis. Con ellos, de alguna forma, se ha construido el pensamiento y la historia de América Latina, por lo que su crisis, querámoslo o no, nos afecta.

El rechazo que la llamada filosofía postmoderna presenta a la Ilustración, es el rechazo al autoritarismo de la razón y al universalismo abstracto de sus principios absolutos e imperativos, confeccionados desde el reino de la razón y desde el escenario espacio-temporal europeo, con desconocimiento, consciente o no, de otras realidades en la historia y en el pensamiento. No obstante, lo que sería la propuesta postmoderna, si es que se puede hablar en esos términos de la Postmodernidad, no reconoce suficientemente el peso específico que las expresiones de la razón, han tenido en la historia concreta de la persona y de los pueblos.

Además, la sociedad postindustrial, contemporánea de la filosofía postmoderna y de alguna forma emparentada con ella, ha producido al neoliberalismo y la globalización, expresiones imperiales y autoritarias establecidas sobre valores absolutos y principios inapelables en el mundo transnacional, que en una $u$ otra forma vivimos y padecemos, a pesar de la deconstrucción, la fragmentación de los paradigmas y el "pensamiento tenue o débil" que proponen los filósofos postmodernos. Así, de esa manera, estamos enfrentados también ante el Doble Rostro de la Postmodernidad. Muchas cosas de la Modernidad deben ser retomadas y cumplidas, como la libertad no realizada; otras, de la Postmodernidad, deben ser asumidas, como el reconocimiento del otro, el respeto a la diferencia, el diálogo de las culturas, la identidad y la diversidad, como formas de la universalidad.
En este marco general de la filosofía mundial contemporánea, creo que el trabajo de los filósofos latinoamericanos y de la filosofía desde América Latina, tiene un papel muy importante que desempeñar para construir una filosofía desde América Latina, lo que significa más que una referencia territorial, una situación en el tiempo, la historia y la cultura, y una determinada perspectiva para enfocar los problemas universales de nuestro tiempo y para lanzar a un horizonte sin fronteras, es decir, universal, los temas tenidos hasta hoy como locales, circunscriptos a una específica historia y geografía. La referencia histórica y cultural no es otra cosa que un observatorio, un punto de vista, con todas las connotaciones e implicancias que esto conlleva.

El proyecto filosófico que enfrente los retos del siglo XXI, proyecto de la unidad en la diversidad, debe superar la separación entre realidad y razón, pues la razón es vida pensada y pensamiento vivido; debe ser entendido, al menos, desde tres puntos de vista o posibilidades: como síntesis, en tanto resultado de una nueva categoría formada por la convergencia de varios afluentes que al dar forma a la unidad resultante, pierden su identidad individual que se disuelve en una dimensión universal; como articulación de diversidades que forman un todo unitario, pero sin perder su particularidad; y como coexistencia de diferentes situaciones que no son asimiladas ni por consenso ni por ninguna formación definida por un grupo hegemónico de poder, sea éste político, social o de otra índole.

Un ejemplo del primer caso podría ser el de los Derechos Humanos; del segundo, el contrato social, el consenso o el proyecto de Estado-Nación; y del tercero, el reconocimiento de los derechos de las minorías, cualquiera sea su naturaleza, en su identidad y expresión particular. Ante las exigencias actuales que enfrentan la filosofía y la ética, proponemos las siguientes hipótesis:

- la filosofía es un proceso dialéctico que va de lo abstracto a lo concreto en la búsqueda de la verdad;

- la historia de la filosofía es una función integradora y relacionadora de los resultados de la filosofía a través del tiempo;

- la filosofía, al buscar lo universal que resulta de las situaciones particulares, es un 
quehacer estrechamente relacionado con el desarrollo histórico y social

- en consecuencia, la filosofía es una tarea de reconstrucción, integración e incorporación entre la vida y el trabajo, el pensamiento y la acción, la ciencia, la moral y el derecho, el análisis y la síntesis.

Como resultado de estas hipótesis formulamos las siguientes proposiciones:

- todo pensamiento, en cuanto acción de la inteligencia y la conciencia, entra en la historia, se historiza;

- el ser humano es un desplegarse que deviene historia. No es una objetividad dada, es movimiento en la historia; no está nunca realizado, pues está realizándose;

- es la forma particular que el movimiento toma en el tiempo a través de la existencia personal o social;

- hay dos elementos que conforman este devenir: la vocación y la voluntad del sujeto. Juntos, vocación y voluntad, forman el ser;

- el proceso de construcción de la historia es el proceso de construcción del ser humano. Éste se crea al crearla;

- lo que el hombre hace forma parte de lo que es; lo que el hombre es forma parte de lo que hace. En este sentido, se entrelazan la ontología y la historia, la filosofía y la práctica;
- el ser, al manifestarse, lo hace históricamente; el propio ser es un manifestarse en la historia;

- la filosofía es la realidad que debe transformarse en concepto; pero es, a la vez, el concepto que debe transformarse en realidad;

- la teoría es la razón de la práctica y ésta la historicidad de la razón. La unidad de ambas es la praxis;

- $\quad$ así entendida, la historia, lejos de ser una deidad implacable, el altar del sacrificio del ser humano, es el tejido de relaciones del actuar del hombre, la esencia misma de un humanismo concreto construido de penas y esperanzas, triunfos y fracasos, ilusiones y decepciones.

La realización de la filosofía como quehacer humano, como diálogo, como compromiso solidario, exige necesariamente asumir posición frente al tema de la globalización y la cultura, reunificar lo disperso, respetar las identidades y proyectarlas al horizonte universal de la razón. Mientras haya preguntas habrá filosofía. Mientras el ser humano sienta la necesidad de explorar el fondo de su conciencia y de su razón, de interrogar al mundo sobre sus contradicciones y de construir y construirse una realidad habitable, la filosofía estará presente ofreciendo desde diferentes ángulos y diversas perspectivas, una forma de construir la historia y de comprender y amar la vida.

\section{REFERÊNCIAS}

BONETE PERALES, Enrique. Aranguren: la ética entre la religión y la política. Madrid: Editorial Tecnos, 1989. VILLORO, Luis. EI pensamiento moderno. Filosofía del Renacimiento. México, DF: Fondo de Cultura Económica, 1992.

ZEA, Leopoldo. Filosofar a la altura del hombre: discrepar para comprender. México, DF: UNAM, 1993. 